\title{
Nonlinear Multigrid for Reservoir Simulation
}

Christensen, Max la Cour; Eskildsen, Klaus Langgren ; Engsig-Karup, Allan Peter; Wakefield, Mark

Publication date:

2012

Document Version

Publisher's PDF, also known as Version of record

Link back to DTU Orbit

Citation (APA):

Christensen, M. L. C., Eskildsen, K. L., Engsig-Karup, A. P., \& Wakefield, M. (2012). Nonlinear Multigrid for Reservoir Simulation. Poster session presented at SIAM SPE Conference on Mathematical Methods in Fluid Dynamics and Simulation of Giant Oil and Gas Reservoirs, Istanbul, Turkey.

\section{General rights}

Copyright and moral rights for the publications made accessible in the public portal are retained by the authors and/or other copyright owners and it is a condition of accessing publications that users recognise and abide by the legal requirements associated with these rights.

- Users may download and print one copy of any publication from the public portal for the purpose of private study or research.

- You may not further distribute the material or use it for any profit-making activity or commercial gain

- You may freely distribute the URL identifying the publication in the public portal

If you believe that this document breaches copyright please contact us providing details, and we will remove access to the work immediately and investigate your claim 


\title{
Nonlinear Multigrid for Reservoir Simulation
}

\author{
Max la Cour Christensen*, Klaus Langgren Eskildsen*, Allan P. Engsig-Karup*, Mark Wakefield** \\ ${ }^{*}$ Technical University of Denmark (DTU), ${ }^{* *}$ Schlumberger
}

\section{Motivation}

In the pursuit of higher resolution simulation models that use all seismic, geological, and dynamic reservoir data - and to make use of modern parallel computing architectures - we consider alternative numerical methods to solve the system of equations governing subsurface porous media flow.

It is standard in conventional techniques to use a globa linearization in a Newton-type method to solve the strongly nonlinear system of equations arising from the spatial and temporal discretization of the governing system of PDEs. Consequently, the memory requirement to store the sparse Jacobian is significant. Such very large linear systems result in the linear solver component to constitute more than $70 \%$ of the computation time in reservoir simulators. Iterative linear solvers depend on effective preconditioners, which can be hard to parallelize to the extent required by many-core simulations. In a first step, we investigate feasibility of using the locally linearizing nonlinear multigrid method Full Approximation Scheme (FAS) in serial to establish algorithmic performance.

\section{Contribution}

- Reservoir simulator based on the nonlinear multigrid method FAS.

- Comparison with conventional techniques in reservoir simulation, specifically a reservoir simulator based on global linearization in Newton's method. Linear solver is FGMRES with CPR-AMG preconditioning implemented using PETSc/BoomerAMG.

\section{Full Approximation Scheme}

Consider the nonlinear system

$$
\mathbf{A}(\mathbf{u})=\mathbf{f},
$$

with error $\mathbf{e}=\mathbf{u}-\mathbf{v}$, where $\mathbf{v}$ is an approximation to the exact solution $\mathbf{u}$ The residual is

$$
\mathbf{r}=\mathbf{f}-\mathbf{A}(\mathbf{v})
$$

Inserting (1) in (2) gives

$$
\mathbf{A}(\mathbf{u})-\mathbf{A}(\mathbf{v})=\mathbf{r}
$$

Using the error relation, equation (3) becomes

$$
\mathbf{A}(\mathbf{v}+\mathbf{e})-\mathbf{A}(\mathbf{v})=\mathbf{r}
$$

For coarsest grid with mesh size $H=2 h$, where $h$ is the fine grid mesh size, this is

$$
\mathbf{A}_{H}\left(\mathbf{v}_{H}+\mathbf{e}_{H}\right)-\mathbf{A}_{H}\left(\mathbf{v}_{H}\right)=\mathbf{r}_{H}
$$

The coarse grid residual $\mathbf{r}_{H}$ is computed by applying the restriction operator $\boldsymbol{I}_{h}^{H}$ to the fine grid residual

$$
\mathbf{r}_{H}=I_{h}^{H} \mathbf{r}_{h}=I_{h}^{H}\left(\mathbf{f}_{h}-\mathbf{A}_{h}\left(\mathbf{v}_{h}\right)\right)
$$

Similarly, the coarse grid approximation $\mathbf{v}_{H}$ is the restriction of the fine grid approximation $\mathbf{v}_{h}$. Using these definitions, equation (4) is rewritten into

$$
\mathbf{A}_{H} \underbrace{\left(I_{h}^{H} \mathbf{v}_{h}+\mathbf{e}_{H}\right)}_{\mathbf{u}_{H}}=\underbrace{\mathbf{A}_{H}\left(I_{h}^{H} \mathbf{v}_{h}\right)+I_{h}^{H}\left(\mathbf{f}_{h}-\mathbf{A}_{h}\left(\mathbf{v}_{h}\right)\right)}_{\mathbf{f}_{H}}
$$

Based on this solution, the coarse grid correction term is computed as $\mathbf{e}_{H}=\mathbf{u}_{H}-I_{h}^{H} \mathbf{v}_{h}$. This correction term is prolongated to a finer grid, where it is used to correct the solution to the residual equation at that grid, which again can be used to determine a correction term for an even finer grid and etc.

The FAS concept is depicted below

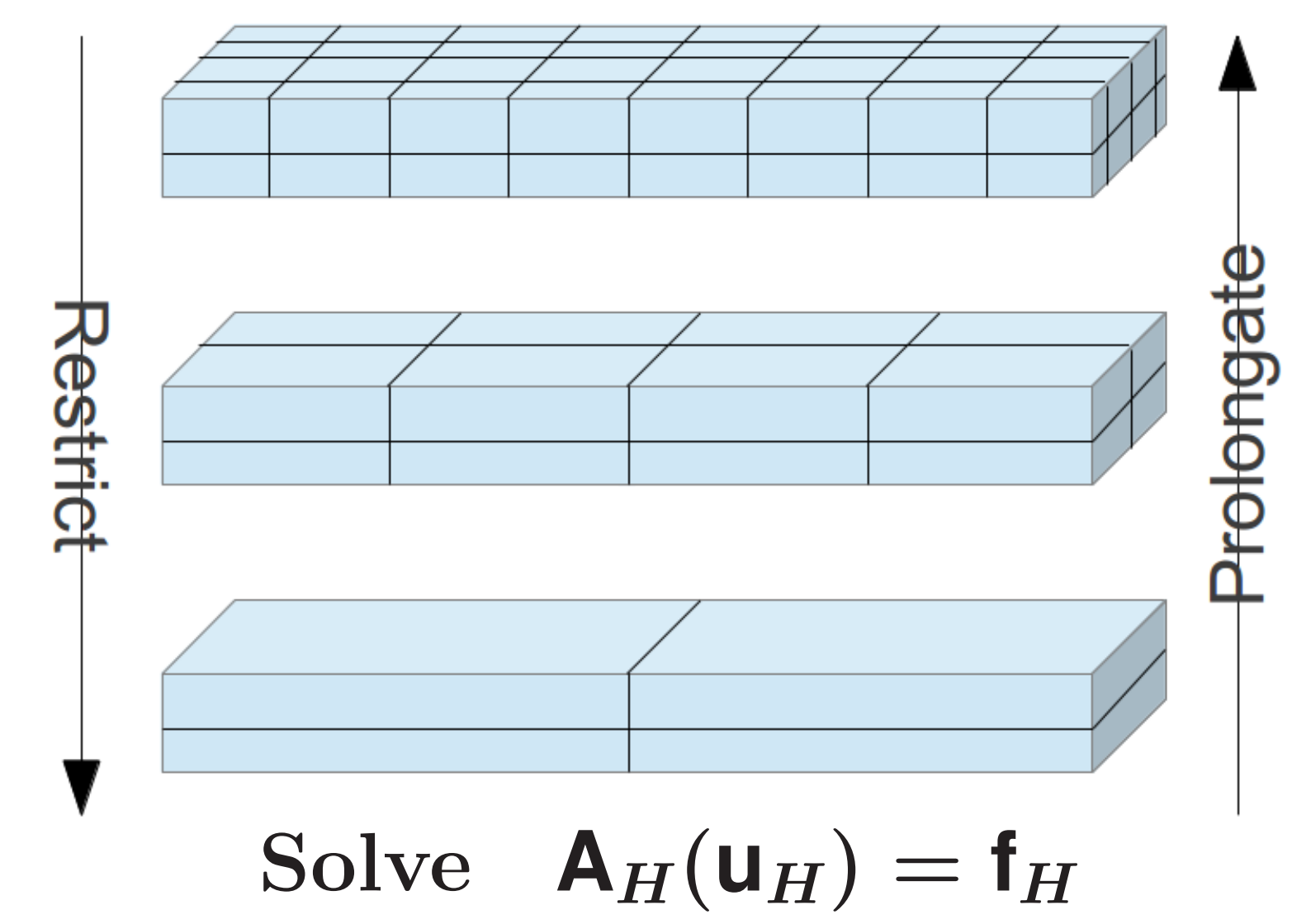

\section{Model equations}

Conservation of mass

$$
\frac{\partial\left(\phi m_{c}\right)}{\partial t}+\nabla \cdot \mathbf{f}^{\alpha}=0
$$

where $\phi$ is the porosity dependent on pressure, $\boldsymbol{m}_{c}$ is molar density of component $c$ and $\mathbf{f}^{\alpha}=b^{\alpha} \mathbf{v}^{\alpha}$ is the flux with the phase molar density $b^{\alpha}$ and the phase velocity $\mathbf{v}^{\alpha}$ given by Darcy's law.

Volume balance constraint

$$
\sum_{\alpha} S^{\alpha}=1
$$

where $S^{\alpha}$ is the saturation of phase $\alpha$

3D with gravitational effects.

- Three immiscible phases: oil, gas and water.

- No capillary effects.

\section{Discretization}

Finite Volume method.

- Backward Euler - Fully Implicit.

\section{FAS components}

- V-cycles.

Nonlinear collective Gauss-Seidel $z$-line smoother using 1 newton iteration.

Thomas algorithm for block tridiagonal systems in smoother.

$\checkmark(x, y)$-semicoarsening, meaning only $x$ - and $\boldsymbol{y}$-directions are coarsened.

\section{Numerical results}

For fixed pattern permeability fields as depicted in Figure 1

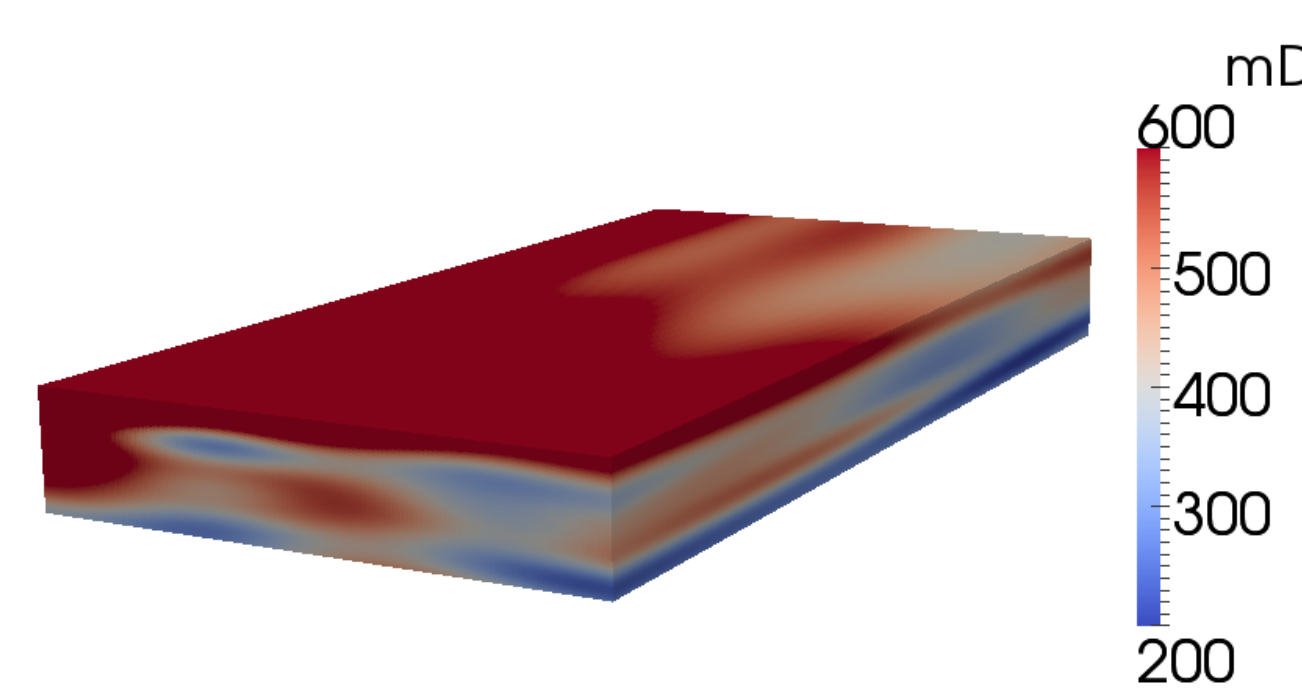

Figure 1: Illustration of permeability field

comparison between a reservoir simulator based on conventional techniques (standard newton(SN)) with global linearization in Newton's method and the simulator based on FAS is displayed in Figure 2. Tests are "gravity inversion", where water and gas components switch place.

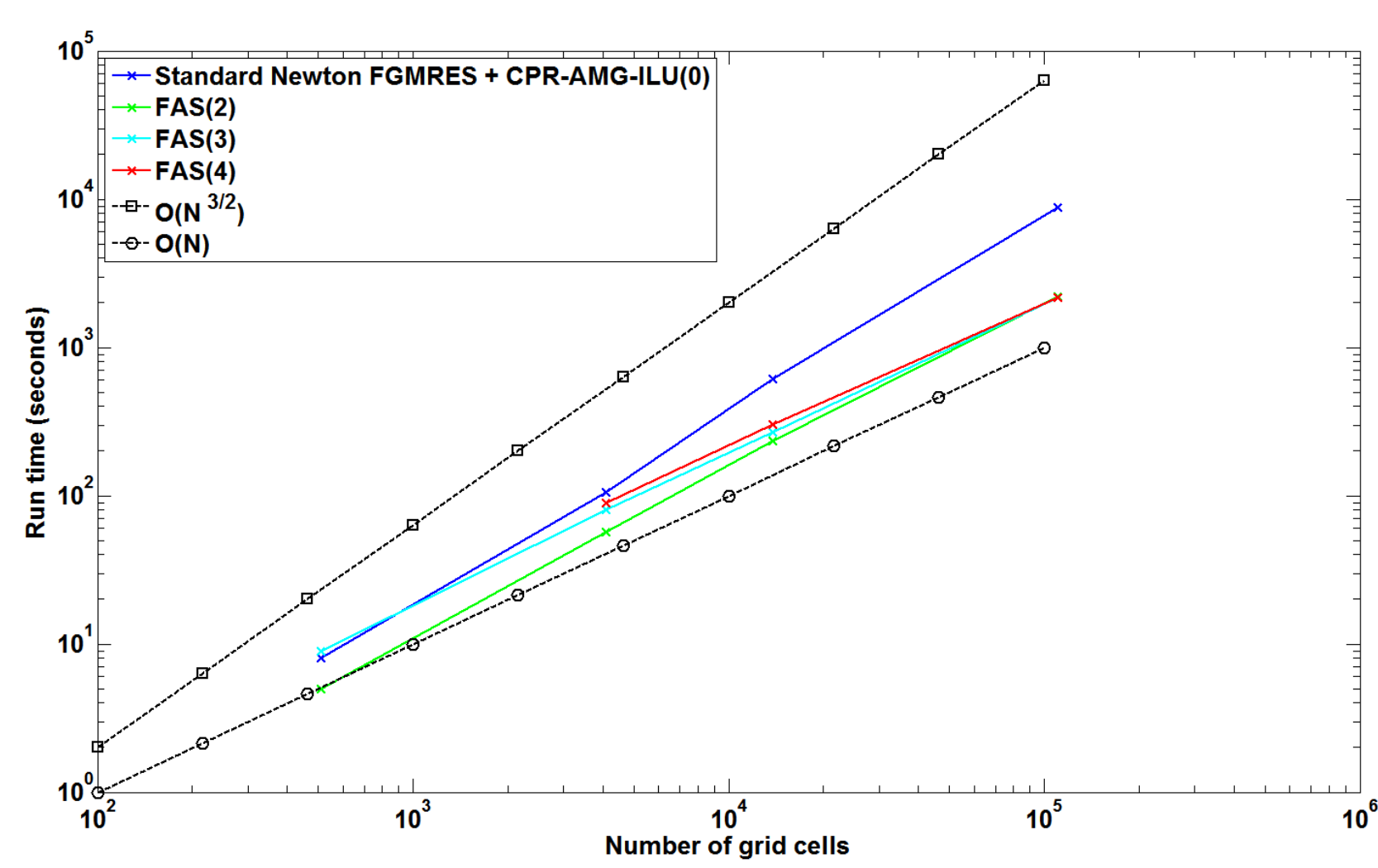

Figure 2: Run time as a function of number of grid cells for standard newton and FAS with 2, 3 and 4 grid levels. Fixed reservoir size: $480 m \times 240 m \times 48 m$.

FAS is faster and appears to have linear scaling for the larger problem sizes. The corresponding number of time steps is listed in Table 1.

\begin{tabular}{c|cccc} 
& \multicolumn{5}{|c}{ Problem size } \\
Method & $8 \times 8 \times 8$ & $16 \times 16 \times 16$ & $24 \times 24 \times 24$ & $48 \times 48 \times 48$ \\
\hline SN & 68 & 101 & 152 & 201 \\
FAS(2) & 40 & 60 & 91 & 171 \\
FAS(3) & 65 & 83 & 82 & 161 \\
FAS(4) & N/A & 93 & 94 & 127
\end{tabular}

Table 1: Number of time steps for the simulations displayed in Figure 2. An adaptive time stepping strategy is employed that seeks to minimize the overall computational effort.

\section{Residuals for outer iteration}

Figure 3 shows residual reduction for 50 time steps, where each line represents a time step.

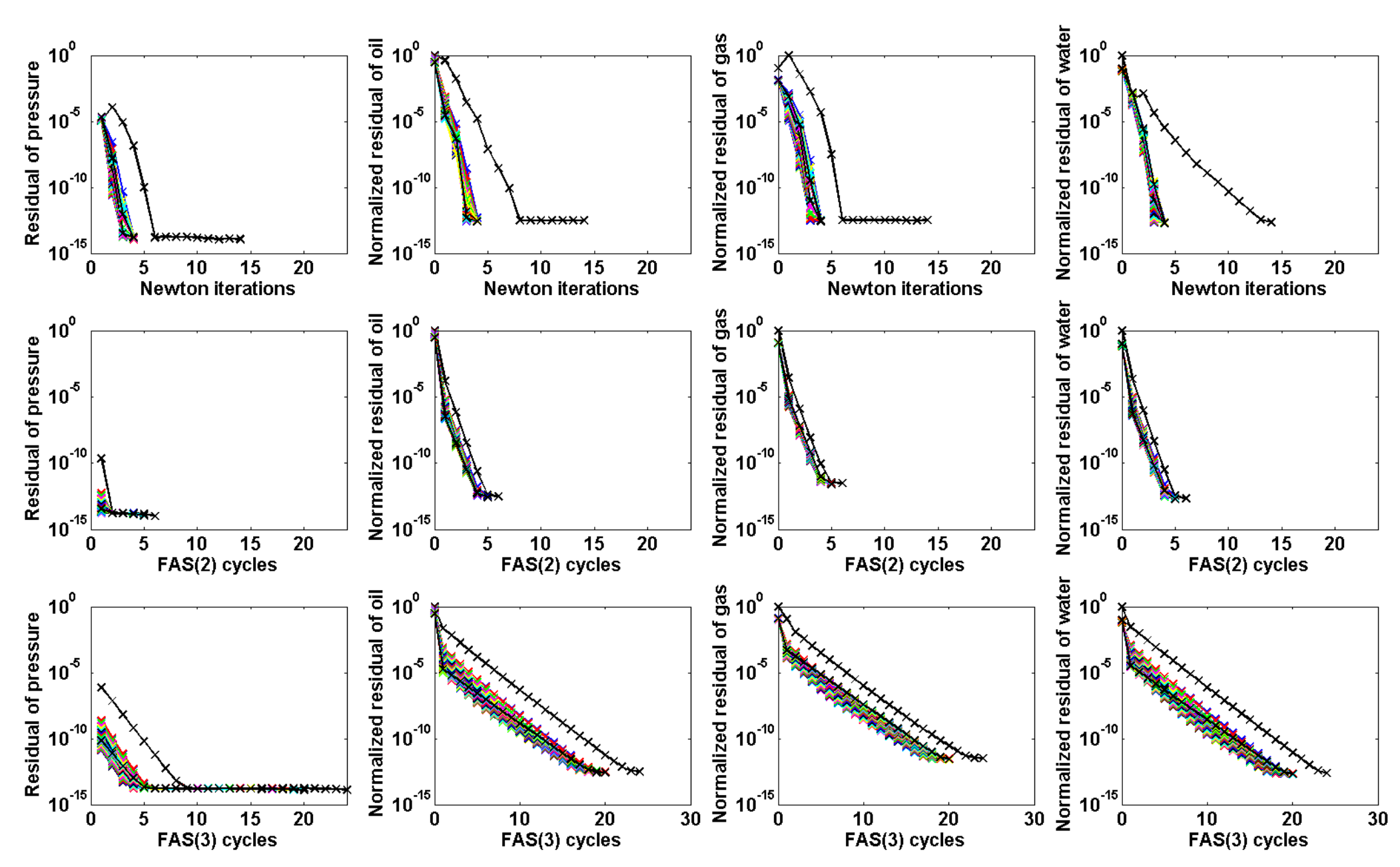

Figure 3: Residual reduction for standard newton and FAS with 2 and 3 grid levels. Problem size: $24 \times 24 \times 24$

FAS has quick initial residual reduction suitable for engineering accuracy purposes.

\section{Computational distribution}

As indicated by Figure 4, the majority of the computational work can be kept in the parallelizable smoother by increasing the number of grid levels in FAS

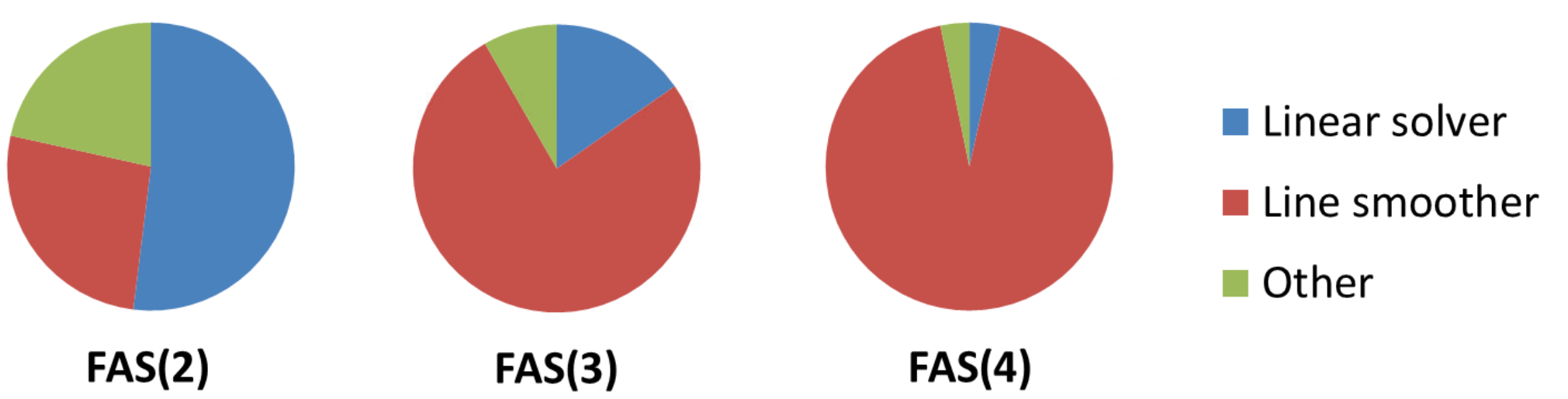

Figure 4: Distribution of computational work for FAS with 2,3 and 4 grid levels. Problem size: $24 \times 24 \times 24$.

\section{Memory comparisons}

FAS is based on local linearization, meaning we avoid having to assemble the Jacobian on the finest grid, which results in significant memory savings as outlined in Table 2.

$\begin{array}{cc}\text { Method: } & \text { kB per grid cell: } \\ \text { Standard newton } & 2.3 \\ \text { FAS(2) } & 0.7 \\ \text { FAS(3) } & 0.3 \\ \text { FAS(4) } & 0.2\end{array}$

Table 2: Memory comparisons for a $128 \times 128 \times 128$ grid cell problem.

\section{Heterogeneity stress test}

As demonstrated by Figure 5, FAS appears to handle very heterogeneous permeability fields better than standard newton.
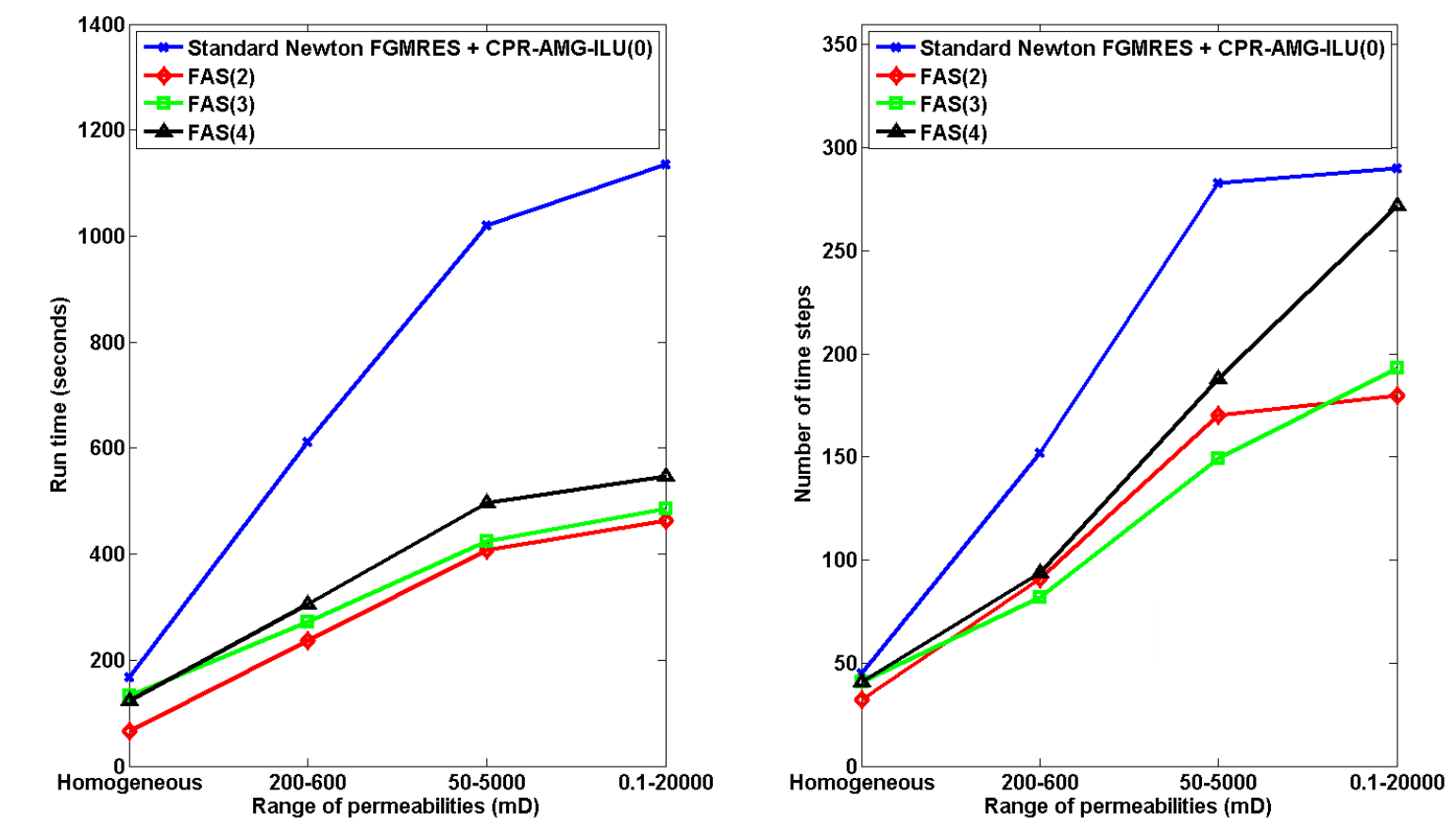

Figure 5: Run time and number of time steps for problems with different ranges of permeability. Problem size: $24 \times 24 \times 24$.

\section{Conclusion and perspectives}

- Promising aspects for modern many-core implementation, e.g. GPUs or Intel MICs.

- Fit larger problems on same hardware and extend to distributed large-scale simulations.

Algorithmic performance established for model equations.

Next step: More complicated model including wells, parallel implementation and feasibility study of polynomial smoothing. 